\title{
Chapter 20 \\ Patients with Rare Head Neck Cancers: \\ Do They Need a Different Approach?
}

\author{
Carla M. L. van Herpen
}

\section{Introduction on Rare tumors}

\section{Definition and Frequency of Rare Cancers}

Rare cancers are the rare diseases in oncology needing specific approaches by the cancer community and national health systems [1]. Rare cancers are defined as malignancies whose incidence is less than 6 per 100,000 inhabitants. The reason why the definition is based on incidence and not on prevalence is, among others, that incidence does not change on other factors than frequency, i.e. not on survival. At this moment rare molecular subgroups of common cancers are not included in the rare cancers list. In absolute numbers more than 500,000 patients per year are diagnosed with a rare cancer, and 4,300,000 rare cancer patients are prevalent in Europe. The definition is widely adopted among the different scientific international societies like the European Society of Medical Oncology (ESMO) and the European Society for Radiotherapy and Oncology (ESTRO). This means that 22\% of all diagnosed cancers are rare and out of the 260 cancer types identified [2], 223 $(86 \%)$ are rare. The European Network for Rare Solid Cancers (EURACAN) uses this definition to create a reference network in order to improve rare cancer care (Table 20.1).

C. M. L. van Herpen $(\bowtie)$

Department of Medical Oncology, Radboud University Medical Center,

Nijmegen, The Netherlands

e-mail: carla.vanherpen@ radboudumc.nl 
Table 20.1 Rare cancers in the head and neck. The rate is the incidence per 100,000 inhabitants per year in Europe [2]

\begin{tabular}{l|l}
\hline Tumor & Rate \\
\hline Nasal cavity and sinuses & 0.44 \\
\hline Nasopharynx & 0.44 \\
\hline Salivary glands major/minor & 1.31 \\
\hline Epithelial cancers of Hypopharynx & 1.19 \\
\hline Larynx & 4.64 \\
\hline Oropharynx & 2.58 \\
\hline Oral cavity & 3.28 \\
\hline Lip & 1.22 \\
\hline Soft tissue sarcoma & 0.29 \\
\hline Bone sarcoma & $(0.8)^{\mathrm{a}}$ \\
\hline Merkel cell & $(0.13)^{\mathrm{a}}$ \\
\hline
\end{tabular}

a The mentioned incidences of bone sarcoma and Merkel cell carcinoma is in the whole body and not exclusive in the head and neck region

\section{Poor Prognosis of Rare Cancers}

In Europe rare cancer patients have poorer survival as compared to common cancer patients. In 2011 the 5-year overall survival of rare cancers was $49 \%$ versus $63 \%$ in common cancers [3].

Moreover, the survival of rare cancer patients in the Netherlands has barely increased over time (from 50\% in 1995-2000 to 56\% in 2012-2016), in contrast to the common cancers (from 59\% in 1995-2012 to $72 \%$ in 2012-2016) [4].

\section{Problems and Challenges in Rare Cancers}

Common challenges posed in rare cancers are a late or incorrect diagnosis [5], less experience and a limited number of experts in the field, limited number of clinical studies (i.e., a limited number of phase III studies), less registered medications, a limited number of guidelines and less registries and biobanks. Patients suffering from rare cancers report higher levels of distress compared to common cancer patients due to increased anxiety and uncertainty correlated with the delayed diagnosis and feelings of isolation invoked by limited disease specific support systems [6]. 


\section{Rare Cancers in the Head and Neck Region}

Rare cancers of the head and neck are epithelial cancers of the larynx, hypopharynx, nasal cavity and sinuses, nasopharynx, major salivary glands and salivary-gland type tumors, oropharynx, oral cavity and lip, eye and adnexa and the middle ear. Besides these malignancies specific for the head and neck region also other rare malignancies can be located in the head and neck region, such as soft tissue sarcoma, bone sarcoma and Merkel cell carcinoma.

Phase III studies performed in the head and neck cancer field frequently include the epithelial cancers of the larynx, hypopharynx, oropharynx and oral cavity together, thereby making them 'less rare'.

\section{Salivary Gland Cancers}

Salivary gland cancer (SGC) is a distinct but heterogeneous group of malignancies comprising approximately $6.5 \%$ of cases within head and neck malignancies. This makes it a rare cancer, with an estimated age-standardized annual incidence of less than 2/100,000 in most countries. The most recent World Health Organization (WHO) classification of Head and Neck Tumors distinguishes 22 histopathological subtypes of SGC, which makes each subtype even rarer. Recognition of, and differentiation between these different subtypes is notoriously difficult and different subtypes exhibit different clinical features adding up to the complexity of the disease. For localized and resectable disease, surgical resection with or without postoperative radiotherapy is the cornerstone of treatment. In case of local recurrent or metastatic $(\mathrm{R} / \mathrm{M})$ disease, systemic treatment is challenging, but urgent given the prognosis of this disease stage. Lumped for all types of SGC with distant metastases ( $71 \%$ of the patients will develop recurrent/metastatic disease) the median overall survival is 15 months with overall survival rates at 1,3 and 5 years of $54.5 \%, 28.4 \%$ and $14.8 \%$, respectively. This, however, varies widely between different subtypes. For example, in adenoid cystic carcinoma median overall survival of several years in patients with distant metastases has been reported. This contrasts with salivary duct carcinoma (SDC), an aggressive subtype of SGC, in which median overall survival for $\mathrm{R} / \mathrm{M}$ disease receiving best supportive care was only 5 months in the past [7].

The clinicopathological diversity of the disease justifies therapy tailored to the specific SGC subtype, highlighting the importance of adequate pathological examination (e.g. subtype, stage, growth pattern), preferably performed by a salivary gland expert pathologist. However, rarity of SGC and its extensive heterogeneity 
hinders large-scale patient accrual in prospective trials and difficulties in correct histopathological subtyping of SGC endanger homogeneity of cohorts. Therefore, performance of clinical trials in SGC is challenging. This is reflected in the limited amount of studies performed with classical chemotherapeutic agents, targeted agents or immunotherapy in SGC.

Survival rates and limited benefit of chemotherapy emphasize that there is an unmet need for new therapeutic strategies for patients with R/M SGC. The paucity of treatment options may be reduced by mapping tumor characteristics and unraveling genetic aberrations in search for possible targets for systemic therapies. By doing so, SGC patients could also share in the benefits of the therapeutic advances made in more common malignancies, especially since the body of evidence for presence of such targets in different histological subtypes is increasing.

\section{Salivary Duct Cancer (as Example)}

SDC is an aggressive subtype of SGC, representing $4-10 \%$ of all SGCs. Overall survival at 3,5 and 10-years is poor: $70.5 \%, 43 \%$ and $26 \%$, respectively. Of the patients with SDC treated with curative intent, 54\% will develop locoregional recurrences and/or distant metastases. In patients with distant metastases, spread to lungs $(54 \%)$ and bones $(46 \%)$ is seen most, and a remarkably high percentage of brain metastasis has been observed (18\%). Given the dismal prognosis and high prevalence of distant metastasis, systemic therapy is often required.

The androgen receptor (AR) and the HER2 receptor (encoded by the HER2 gene) are frequently expressed in SDC, respectively in 78-96\% and 29-46\% of cases. Targeting AR and/or HER2 is promising and are the best studied therapies in SDC patients.

A prospective phase 2 trial evaluating the effect of combined androgen blockade (CAB) with leuprorelin acetate and bicalutamide in 36 SGC patients (of which 34 were SDC patients), showed partial or complete responses in $41.7 \%$ [95\%-CI 25.5-59.2\%] and stabilization of disease in 44.4\% [95\%-CI 27.9-61.9]. The median progression-free survival was 8.8 months [95\% CI, 6.3-12.3 months] and the median overall survival was 30.5 months [95\% CI, 16.8 months to not reached] [8]. Especially given the low rate of observed grade 3 or 4 toxicity, CAB plays an important role in the palliative treatment of AR positive SDC patients. Besides its role in palliative treatment, androgen deprivation therapy (ADT) may also be beneficial in the adjuvant setting. Based on retrospective data, adjuvant ADT results in significantly improved 3-year disease free survival (DFS) in patients with stage 4A AR-positive SDC $\quad(48.2 \% \quad[95 \%$-CI $14.0-82.4 \%]$ versus $27.7 \% \quad$ [95\%-CI $18.5-36.9 \%$ ] in the control group who did not receive adjuvant ADT). Differences in overall survival were just below and above significance level, depending on whether or not correction for confounders was performed [9]. 
Trastuzumab in combination with taxane based chemotherapy is the best studied combination on HER-2 targeted therapy. Fifty-seven eligible patients with SDC were enrolled in a phase II study. The overall response rate was $70.2 \%$ (95\% CI, $56.6 \%$ to $81.6 \%$ ), and the clinical benefit rate was $84.2 \%$ (95\% CI, $72.1 \%$ to $92.5 \%$ ). Median progression-free and overall survival times were 8.9 months (95\% CI, 7.8 to 9.9 months) and 39.7 months (95\% CI, not reached), respectively [10]. This combination could potentially be amplified with the addition of another agent targeting HER2 (e.g. pertuzumab, lapatinib) or after progressive disease replacing trastuzumab with the antibody-drug conjugate trastuzumab-emtansine. A oral presentation at the American Society of Clinical Oncology (ASCO) in 2019 emphasizes the potential of trastuzumab-emtansine in HER2-amplified SGC, as 9 out of 10 patients (0-3 lines of prior treatment, median of 2) responded on this treatment. Presumably most of these patients were SDC patients. Median PFS was not reached after a median follow-up of 12 months [11]. In analogy with the positive results achieved in HER2 positive breast cancer by adding pertuzumab to docetaxel/trastuzumab and the cases reported on this combination in SDC, this triple combination deserves a continuation in clinical studies in SDC.

Targeting HER2 in SDC patients with HER2 overexpression is thus promising. In patients co-expressing AR and HER2 it is yet unclear whether therapy targeting AR or HER2 is the best approach. However, in case of extensive or rapidly progressive disease, HER2 targeting therapy in combination with taxane-based chemotherapy is the preferable choice over ADT.

Besides AR and HER2, a wide spectrum of mutations, is observed in lower frequencies in SDC, which altogether forms a genetic landscape highly similar to apocrine breast cancer. This includes mutations in TP53 (53-68\%), PIK3CA (18-26\%), HRAS (16-23\%), BRAF (4\%) and AKT1 (1.5\%). Reports on the use of drugs aiming at these targets in clinical practice are scarce [12].

In summary, SDC has many targets amenable for systemic therapy. Elaborate mapping of tumor characteristics regarding receptor expression, genomics and pathway alterations are key to alter the dismal prognosis of patients with locally advanced or metastatic SDC.

\section{NTRK Inhibition}

Secretory carcinoma (SC), previously named mammary analogue secretory carcinoma (MASC), is a relatively new entity that was first described in the salivary glands in 2010 [13]. In retrospect, most cases of what is now called SC were initially classified as acinic cell carcinoma and also as polymorphous adenocarcinoma or adenocarcinoma NOS. SC is rare, is most often found in the parotid gland (58-68\%) and behaves relatively indolent with a good prognosis. R/M disease is rare (estimated 5 and 10 years survival 95\%). The genetic hallmark of SC is a 
ETV6-NTRK3 gene fusion as a result of a t $(12 ; 15)(\mathrm{p} 13 ; \mathrm{q} 25)$ translocation, although other gene fusions with ETV6 have been described (for instance ETV6-MET and ETV6-RET). NTRK gene fusions are known oncogenic drivers and have been described in other tumor types. This ETV6-NTRK3 gene fusion therefore provides a promising target for systemic therapy, and the body of evidence for efficacy of TRK-inhibitors (e.g. larotrectinib, entrectinib, repotrectinib, LOXO-195) in patients with NTRK gene fusions is expanding. A recent phase II trial evaluating the efficacy of larotrectinib in NTRK fusion positive patients included 12 patients with recurrent or metastatic (MA)SC and reported a response rate of $75 \%$ and the median progression free survival was not reached after median follow-up of 9.9 months [14]. Responses in patients with (MA)SC have also been observed for entrectinib and repotrectinib. A phase I/II trial evaluating LOXO-195 in second line is currently recruiting and is open for inclusion of NTRK fusion positive SGC patients previously treated with a TRK-inhibitor (NCT03215511).

Whether NTRK gene fusions are present in other subtypes of SGC is currently unknown, but treatment with TRK-inhibitors is a very promising treatment option, an option that should be investigated in all SGC patients with advanced disease.

\section{Future}

Patients with rare cancers deserve a different approach. Only then we can make progress to improve care and cure in rare cancers.

In the rare cancer agenda 2030 written by the Joint action on Rare Cancers (JARC) ten recommendations are made [1]. In rare cancers networking is crucial; this means that networking with patients, in health care systems, but also in medical education is extremely important. Furthermore, regulation on rare cancers should tolerate a higher degree of uncertainty, which means that the 'rules' we made for registration of new medications in common cancers (mostly based on phase III evidence) cannot be the same for rare cancers.

\section{References}

1. Rare Cancer Agenda 2030. JARC 2016-2019.

2. www.rarecare

3. Gatta et al. Eur J Cancer 2011; 47:2493.

4. IKNL Kankerzorg in beeld: zeldzame kanker Nov 2017.

5. Ray-Coquard et al. Ann Oncol. 2012.

6. Bergerot CD, Bergerot PG, Philip EJ, et al. Assessment of distress and quality of life in rare cancers. Psycho-Oncology. 2018;27(12):2740-6. https://doi.org/10.1002/pon.4873.

7. Lassche G, van Boxtel W, Ligtenberg MJL, van Engen-van Grunsven ACH, van Herpen CML. Advances and challenges in precision medicine in salivary gland cancer. Cancer Treat Rev. 2019;80:101906. https://doi.org/10.1016/j.ctrv.2019.101906. 
8. Fushimi C, Tada Y, Takahashi H, Nagao T, Ojiri H, Masubuchi T, et al. A prospective phase II study of combined androgen blockade in patients with androgen receptor-positive metastatic or locally advanced unresectable salivary gland carcinoma. Ann Oncol. 2018;29:979-84.

9. van Boxtel W, Locati LD, van Engen-van Grunsven ACH, Bergamini C, Jonker MA, Fiets E, et al. Adjuvant androgen deprivation therapy for poor-risk, androgen receptor-positive salivary duct carcinoma. Eur J Cancer. 2019;110:62-70.

10. Takahashi H, Tada Y, Saotome T, Akazawa K, Ojiri H, Fushimi C, et al. Phase II trial of trastuzumab and docetaxel in patients with human epidermal growth factor receptor 2-positive salivary duct carcinoma. J Clin Oncol. 2019;37:125-34.

11. Li BT, Shen R, Offin M, Buonocore DJ, Myers ML, Venkatesh A, et al. Ado-trastuzumab emtansine in patients with HER2 amplified salivary gland cancers (SGCs): results from a phase II basket trial. J Clin Oncol. 2019;37:6001.

12. Uijen MJM, Lassche G, van Engen-van Grunsven ACH, Tada Y, Verhaegh GW, Schalken JA, Driessen CML, van Herpen CML. Systemic therapy in the management of recurrent or metastatic salivary duct carcinoma: a systematic review (submitted).

13. Skalova A, Vanecek T, Sima R, Laco J, Weinreb I, Perez-Ordonez B, et al. Mammary analogue secretory carcinoma of salivary glands, containing the ETV6-NTRK3 fusion gene: a hitherto undescribed salivary gland tumor entity. Am J Surg Pathol. 2010;34:599-608.

14. Drilon A, Laetsch TW, Kummar S, DuBois SG, Lassen UN, Demetri GD, et al. Efficacy of larotrectinib in TRK fusion-positive cancers in adults and children. $\mathrm{N}$ Engl $\mathrm{J}$ Med. 2018;378:731-9.

Open Access This chapter is licensed under the terms of the Creative Commons Attribution 4.0 International License (http://creativecommons.org/licenses/by/4.0/), which permits use, sharing, adaptation, distribution and reproduction in any medium or format, as long as you give appropriate credit to the original author(s) and the source, provide a link to the Creative Commons license and indicate if changes were made.

The images or other third party material in this chapter are included in the chapter's Creative Commons license, unless indicated otherwise in a credit line to the material. If material is not included in the chapter's Creative Commons license and your intended use is not permitted by statutory regulation or exceeds the permitted use, you will need to obtain permission directly from the copyright holder.

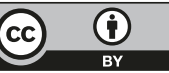

